\title{
Stochastic sandpile model on small-world networks: scaling and crossover
}

\author{
Himangsu Bhaumik, S. B. Santra \\ Department of Physics, Indian Institute of Technology Guwahati, Guwahati-781039, Assam, India.
}

\begin{abstract}
A dissipative stochastic sandpile model is constructed on one and two dimensional smallworld networks with different shortcut densities $\phi$ where $\phi=0$ and 1 represent a regular lattice and a random network respectively. In the small-world regime $\left(2^{-12} \leq \phi \leq 0.1\right)$, the critical behaviour of the model is explored studying different geometrical properties of the avalanches as a function of avalanche size $s$. For both the dimensions, three regions of $s$, separated by two crossover sizes $s_{1}$ and $s_{2}\left(s_{1}<s_{2}\right)$, are identified analyzing the scaling behaviour of average height and area of the toppling surface associated with an avalanche. It is found that avalanches of size $s<s_{1}$ are compact and follow Manna scaling on the regular lattice whereas the avalanches with size $s>s_{1}$ are sparse as they are on network and follow mean-field scaling. Coexistence of different scaling forms in the small-world regime leads to violation of usual finite-size scaling, in contrary to the fact that the model follows the same on the regular lattice as well as on the random network independently. Simultaneous appearance of multiple scaling forms are characterized by developing a coexistence scaling theory. As SWN evolves from regular lattice to random network, a crossover from diffusive to super-diffusive nature of sand transport is observed and scaling forms of such crossover is developed and verified.
\end{abstract}

Keywords: Self-organized criticality, Sandpile model, Small-World Networks

\section{Introduction}

The spontaneous emergence of spatio-temporal correlation and hence the criticality in many natural phenomena are found to be outcome of self-organized criticality (SOC) [1, 2, 2, 3]. The appearance of SOC on real-world complex networks is ubiquitous in nature. Examples are spreading from Biology to Astrophysics to technology such as, avalanche mode of activity in the neural network of brain [4, 5], earthquake dynamics on the network of faults in the crust of the earth [6], rapid rearrangement of the magnetic field network in the corona [7], propagation of information through a network with a malfunctioning router causing the

Email address: santra@iitg.ac.in (S. B. Santra) 
breakdown of the Internet network [8], breakdown of the electric power grid [9] and many others.

On the other hand, small-world network (SWN) [10] has a very interesting property. It not only interpolates between the regular lattice (RL) and the random network (RN) but also it preserves the properties of both RL and RN, namely high "clustering-coefficient" (concept of neighborhood) and "small-world-effect" (small average shortest distance between any two nodes) respectively. It is always intriguing to study the models of SOC on networks such as SWN. Sandpile, a prototypical model to study SOC on RL introduced by Bak, Tang, and Wiesenfeld (BTW) [11, 12] gives rise to anomalous (multi) scaling [13, 14, 15]. The scaling behaviour of such a deterministic model when studied on RN exhibited very different behaviour than its behaviour on RL [17]. A Mean-Field (MF) [16, 17, 18, 19] scaling behaviour found to describe the avalanche properties rather than an anomalous scaling of BTW model on RL. Stochastic sandpile models (SSM) which incorporates random distribution of sand grains during avalanche, exhibit a scaling behaviour with definite critical exponents that follows finite-size scaling (FSS) when studied on RL. SSM defines a robust universality class called Manna class [20, 21]. Not only SSM shows robust scaling behaviour than the deterministic BTW model, it is also able to explain certain experimentally observed avalanche behaviour [22]. SSM found to be one of the most studied models in various dimensions in SOC literature [23, 24, 25]. However, there are not many studies that report the scaling behaviour of SSM on SWN. It is then important to study a stochastic sandpile model like SSM on SWN and verify whether all such scaling forms would be preserved or not.

In this paper, a dissipative stochastic sandpile model (DSSM) is developed and studied on SWN as a function of shortcut density $\phi$ in one and two dimensions. Dissipation of the sand occurs in the bulk of the system in an annealed manner rather than at the boundary. Various scaling forms of different avalanche properties are identified and numerically verified.

\section{The model: DSSM on SWN}

SWN is generated by adding shortcuts between two randomly chosen sites both of a one dimensional (1d) lattice and those of a two dimensional (2d) square lattice. The shortcut density, number of added shortcuts per existing bond, is defined as $\phi=N_{\phi} /\left(d L^{d}\right)$ where $N_{\phi}$ is the number of shortcuts added and $d L^{d}$ is the number of bonds (without shortcuts) present in a $d$-dimensional lattice of linear size $L$ with periodic boundary conditions (pbc) in both the directions. Care has been taken to avoid self-edges of any node and multi-edges between any two nodes. The system corresponds to a RL as $\phi \rightarrow 0$, whereas in the limit $\phi \rightarrow 1$ it corresponds to a RN. The system behaves like SWN for the intermediate values of $\phi$ such as $2^{-12}$ to $2^{-3}[19,26]$. To study the sandpile dynamics on an SWN, a suitable value of $\phi$ is chosen and the SWN is driven by adding sand grains, one at a time, to randomly chosen nodes. If the height $h_{i}$ of the sand column at the $i$ th node becomes greater than or equal to the predefined threshold value $h_{c}$, which is equal to 2 here, the $i$ th node will topple and the height of the sand column of the $i$ th node will be reduced by $h_{c}$. The toppled two sand grains are then distributed among two of its randomly selected adjacent nodes which 
are connected to the toppled node either by shortcuts or by nearest neighbour bonds. The toppling rule of the $i$ th critical node of DSSM can be written as

$$
\begin{aligned}
h_{i} & \rightarrow h_{i}-h_{c}, \\
\text { and } \quad h_{j} & = \begin{cases}h_{j} & \text { if } r \leq \epsilon_{\phi}, \\
h_{j}+1 & \text { otherwise }\end{cases}
\end{aligned}
$$

where $j$ is two randomly and independently selected adjacent nodes, and $r$ is a random number uniformly distributed between 0 and 1. During transport of sand grains from one node to another, every sand grain is attempted for dissipation from the system with a probability $\epsilon_{\phi}$ to avoid overloading of the system. If the toppling of a node causes some of the adjacent nodes unstable, subsequent toppling follow on these unstable nodes in a cascading manner which leads to an avalanche. An avalanche stops when there is no unstable node present in the system. During an avalanche no sand grain is added to the system to ensure that the system in under slow driving force and at the same time has a fast relaxation, which manifests the separation of time scale, the key ingredient in a SOC system.

The probability of dissipation $\epsilon_{\phi}$ is taken as the inverse of the average number of steps $\left\langle n_{\phi}\right\rangle$ required for a random walker to reach the lattice boundary starting from any lattice point [27, 19]. The dissipation factor $\epsilon_{\phi}=1 /\left\langle n_{\phi}\right\rangle$ for a given $\phi$ is then determined using the numerically estimated values of $\left\langle n_{\phi}\right\rangle$.

\section{Numerical simulations and steady state}

Extensive computer simulations are performed to study the dynamics of DSSM on SWN for various values of $\phi$ and system sizes $L$ both in $1 \mathrm{~d}$ and $2 \mathrm{~d}$. For $1 \mathrm{~d}, L$ is varied from 1024 to 8192 , whereas for $2 \mathrm{~d}, L$ is varied from 128 to 1024 in multiple of 2 . To estimate $\epsilon_{\phi}$, for a given $L$ and $\phi,\left\langle n_{\phi}\right\rangle$ is calculated by performing $2 \times 10^{6}$ random walks in 16 different random configurations of SWN. Knowing $\epsilon_{\phi}$, starting from the empty configuration of $h_{i}$ (i.e., $h_{i}=0 \forall i$ ), sand grains are added at random positions. The average height of the sand column $\langle h\rangle=\sum_{0}^{L^{d}} h_{i} / L^{d}$ for three different values of $\phi$ are plotted against number of avalanches in Figs. 1(a) and 1(b) for $1 \mathrm{~d}$ and $2 \mathrm{~d}$ respectively. It can be observed that after a transient period the system evolves to a steady state which corresponds to equality of current of sand influx (due to adding sand) and current of sand outflux (due to dissipation of sand). Such balance of sand influx and outflux maintains the system in a critical steady state. Critical properties of DSSM on SWN are characterized studying various properties of avalanche like size $s$ (total number of toppling in an avalanche), area $a$ (number of distinct nodes toppled in an avalanche), etc., in the steady state at different values of shortcut density $\phi$. For a given $L$ and $\phi$, data are averaged over $32 \times 10^{6}$ avalanches (collected in the steady state) on 32 different SWN configurations. The information of an avalanche is kept by storing the number of toppling of every node in an array $S_{\phi}[i], i=1, \cdots, L^{d}$ which was set to zero initially. All geometrical properties of an avalanche such as avalanche size $s$, 

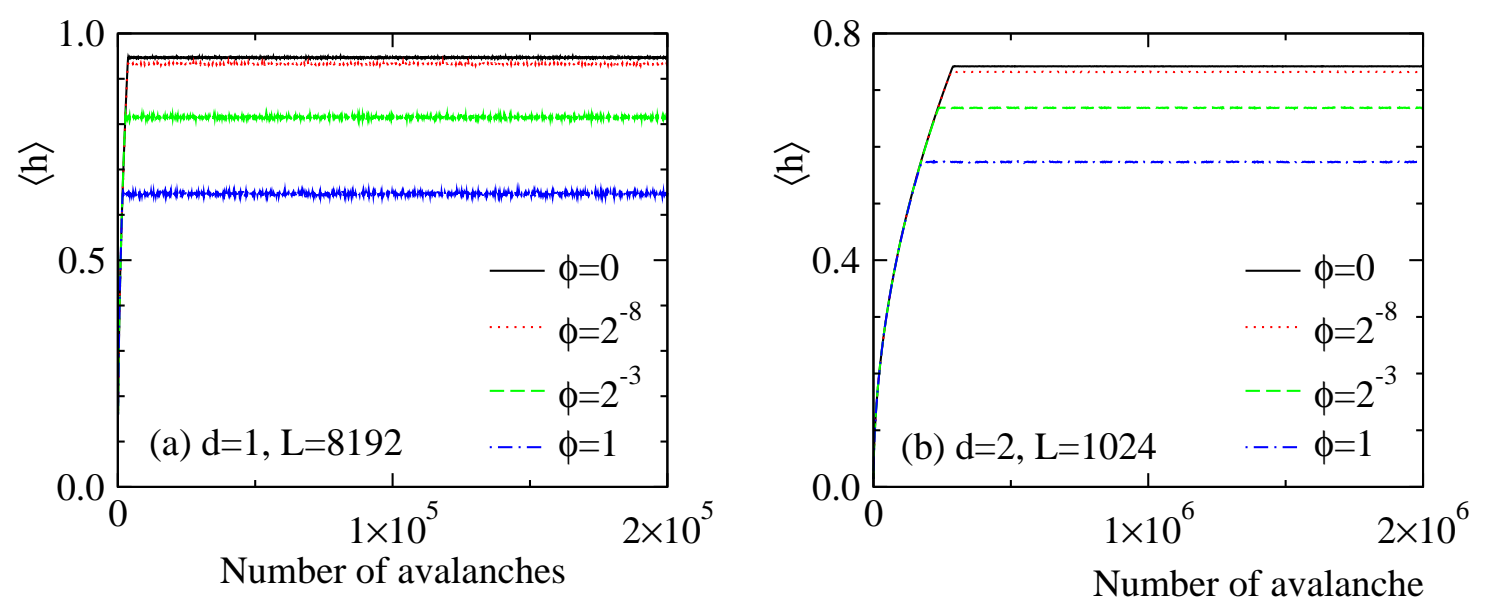

Figure 1: Plot of $\langle h\rangle$ against the number of avalanches for different values of $\phi: 0$ (solid black line) , $2^{-8}$ (dotted red line), $2^{-3}$ (dashed green line), and 1 (blue dotted dashed line) for $1 \mathrm{~d}$ with $L=8192$ in (a), and for 2 d with $L=1024$ in (b).

avalanche area $a$, etc., can be estimated in terms of $S_{\phi}[i]$ as

$$
s=\sum_{i=1}^{L^{d}} S_{\phi}[i], \quad a=\sum_{i=1}^{L^{d}} 1
$$

for all $S_{\phi}[i] \neq 0$.

\section{Results and discussion}

The critical properties of stochastic sandpile model are already known on $\operatorname{RL}(\phi=0)$ [15, 24, 25] as well as on $\mathrm{RN}(\phi=1)$ [28, 29]. In the present study, the critical behaviour of DSSM on small-world regime $\left(2^{-12} \leq \phi \leq 0.1\right)$ will be addressed. Limiting behaviour of such result would confirm the results corresponding to $\phi=0$ and $\phi=1$.

\subsection{Toppling surface: fragmentation, compactness, and fluctuation}

In order to characterize various geometrical properties of avalanche one needs to visualize the avalanche in a suitable parameter space. The values of the toppling number $S_{\phi}[i]$ of an avalanche at different nodes of SWN define a surface called toppling surface [30] which not only serves as an important quantity to visualize an avalanche but also presents important scaling behaviour of several geometrical properties of the avalanche [31, 32]. For an intermediate value of $\phi$ (SWN regime), the toppling surfaces of DSSM for both $1 \mathrm{~d}$ and $2 \mathrm{~d}$ are presented in Fig. 2 for various avalanches of different area and sizes. The upper panel corresponds to the toppling surfaces on $1 \mathrm{~d}$ for $L=256$ and $\phi=2^{-6}$, and the lower panel represents those on a $2 \mathrm{~d}$ square lattice of size $L=256$ and $\phi=2^{-8}$. Since the avalanche clusters are occurring on a network, it might be sparse in the sense that different parts of the same cluster are separated by an Euclidean distance greater than the lattice spacing. A 

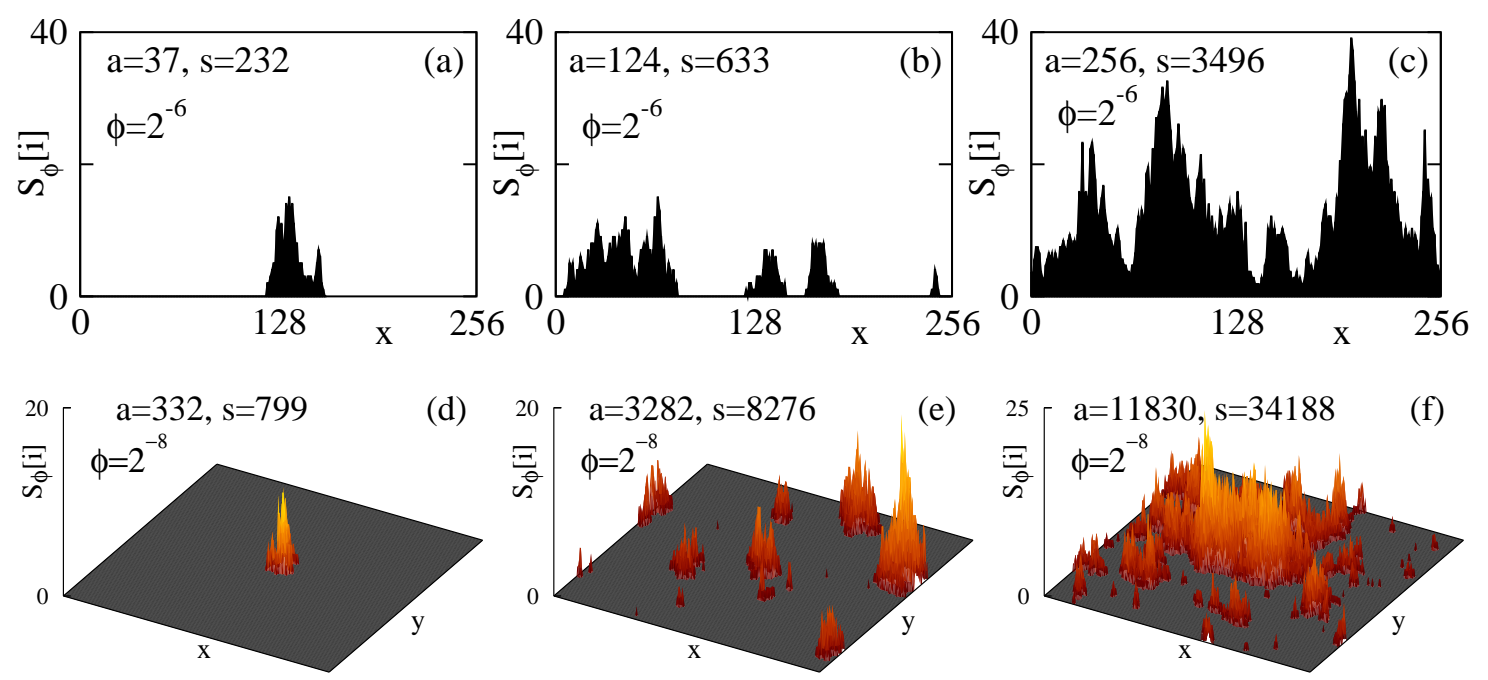

Figure 2: Toppling surfaces of various avalanche clusters of different avalanche area $(a)$ and sizes $(s)$ in the SWN regime are shown. Toppling surfaces on 1d lattice of $L=256$ and $\phi=2^{-6}$ are presented in the upper panel and those on $2 \mathrm{~d}$ square lattice of size $L=256$ and $\phi=2^{-8}$ are presented in the lower panel. The values of $s$ and $a$ are mentioned as legends in the respective plots.

cluster is said to be compact if all the toppled sites are only separated by nearest neighbour lattice spacing. Otherwise the cluster will be called sparse or fragmented. It can be seen that for both $1 \mathrm{~d}$ and $2 \mathrm{~d}$, the avalanches of smaller area are compact as those are confined in a length scale where SWN behaves as a RL. The avalanches of intermediate area and sizes are found to be sparse as those avalanches are exposed to the network. The avalanches of area comparable to system size in $1 \mathrm{~d}$ are also found compact (see Fig,2(b)) whereas those in $2 \mathrm{~d}$ still remain sparse (see Fig,2(f)). Hence, not only the characteristic behaviour of avalanches are different for different values of avalanche size $s$, but also they differ considerably on different dimensions.

As the avalanches are compact single cluster with no fragmentation on RL and are fragmented in several sub-cluster on RN (see Fig. 2), the compactness of an avalanche need to be studied. If an avalanche of size $s$ is fragmented into $N_{f}$ number of fragments with area $a_{f}$ of the $f$ th fragment, then the compactness $\mathcal{C}_{s}$ and the fluctuation in fragment area, $\chi_{s}$, can be defined as

$$
\mathcal{C}_{s}=\left\langle\frac{1}{N_{f}}\right\rangle_{s}, \quad \chi_{s}=\left\langle\left[1-\frac{\left\langle a_{f}\right\rangle^{2}}{\left\langle a_{f}^{2}\right\rangle}\right]\right\rangle_{s}
$$

giving $\mathcal{C}_{s}=1$ and $\chi_{s}=0$ for $N_{f}=1$ (no fragmentation) and $\mathcal{C}_{s} \rightarrow 0$ and $\chi_{s} \rightarrow 1$ for large $N_{f}$. For SWN regime, $\mathcal{C}_{s}$ and $\chi_{s}$ are plotted in Figs. 3(c) and 3(d) for $1 \mathrm{~d}$ and $2 \mathrm{~d}$ respectively. It can be seen that there exists three different regimes of avalanche size $s$ in $1 \mathrm{~d}$ and two such regimes in $2 \mathrm{~d}$ where both $\mathcal{C}_{s}$ and $\chi_{s}$ display different characteristic behaviour with $s$. Such regimes are identified by the dashed lines at the tentative crossover sizes $s_{1}$ and $s_{2}$, marked by crosses in Fig. 3, which will be estimated later. Both in $1 \mathrm{~d}$ and $2 \mathrm{~d}, \mathcal{C}_{s}$ in the region $s_{1}<s<s_{2}$, is quite low, less than 0.20 . Hence an avalanche cluster on an average is 

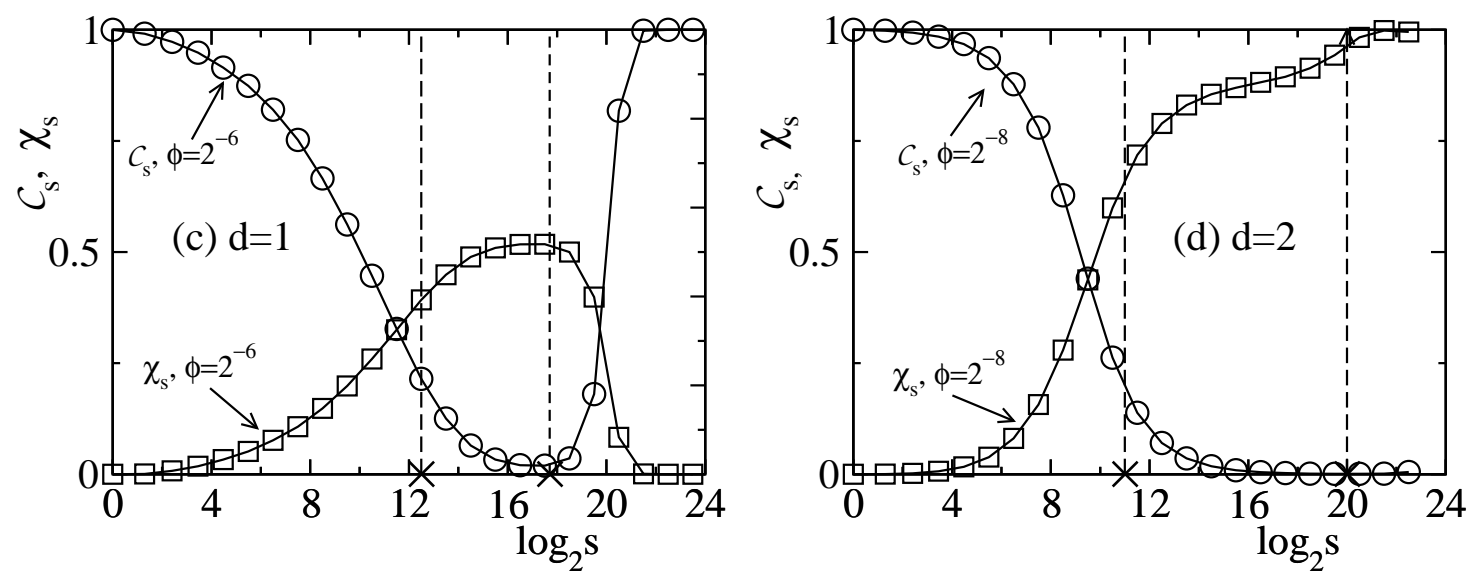

Figure 3: $\quad$ Plot of $\mathcal{C}_{s}(\bigcirc)$ and $\chi_{s}(\square)$ against $s$ in semi-logarithmic scale for $1 \mathrm{~d}$ in (a) and for 2d in (b). Data for $1 \mathrm{~d}$ are collected for $\phi=2^{-6}$ on the system size $L=8192$, whereas, that for $2 \mathrm{~d}$ are collected for $\phi=2^{-8}$ and $L=1024$. Vertical dashed lines in each figure represent tentatively crossover sizes .

fragmented at least into five pieces. These clusters are called sparse. Similarly, $\chi_{s}$ attains a high value and remains more or less constant till $s \approx s_{2}$. For the avalanches of size $s<s_{1}$, $\mathcal{C}_{s}$ is high between 1 and 0.20 indicating a compact avalanche. Consequently, $\chi_{s}$ is small. Whereas for the avalanches of size $s>s_{2}$, the behaviour of $\mathcal{C}_{s}$ and $\chi_{s}$ are very different in $1 \mathrm{~d}$ and $2 \mathrm{~d}$. For $1 \mathrm{~d}, \mathcal{C}_{s}$ starts increasing with $s$ and attains 1 and at the same time $\chi_{s}$ goes to zero. The avalanches then become a single compact avalanche again (see, Fig.2(c)) whereas, in $2 \mathrm{~d}, \mathcal{C}_{s}$ is close to zero and $\chi_{s}$ is almost one. It suggests that in $2 \mathrm{~d}$ avalanches are not only fragmented but also the fragments have high fluctuation in their masses. These are consistence with the toppling surface presented in Fig. 2(f). Usually on RL $(\phi=0)$, the avalanches are trivially found to be compact but on the random network $(\phi=1)$, they are found to be fragmented.

\subsection{Conditional expectation and scaling}

In order to characterize the toppling surface quantitatively, average height $S_{s}$ and area $\left\langle a_{s}\right\rangle$ of the toppling surfaces are studied as a function of the avalanche size $s$. The average area $\left\langle a_{s}\right\rangle$ of an avalanche of fixed size $s$ is defined as

$$
\left\langle a_{s}\right\rangle=\int a P(a \mid s) d s
$$

where $P(a \mid s)$ is the conditional probability for an avalanche of area $a$ and size $s$ to appear [16]. The average height $\left\langle S_{s}\right\rangle$ of a toppling surface is defined as,

$$
S_{s}=\frac{1}{a} \sum_{i=1}^{a} S_{\phi}[i]=\frac{s}{a},
$$



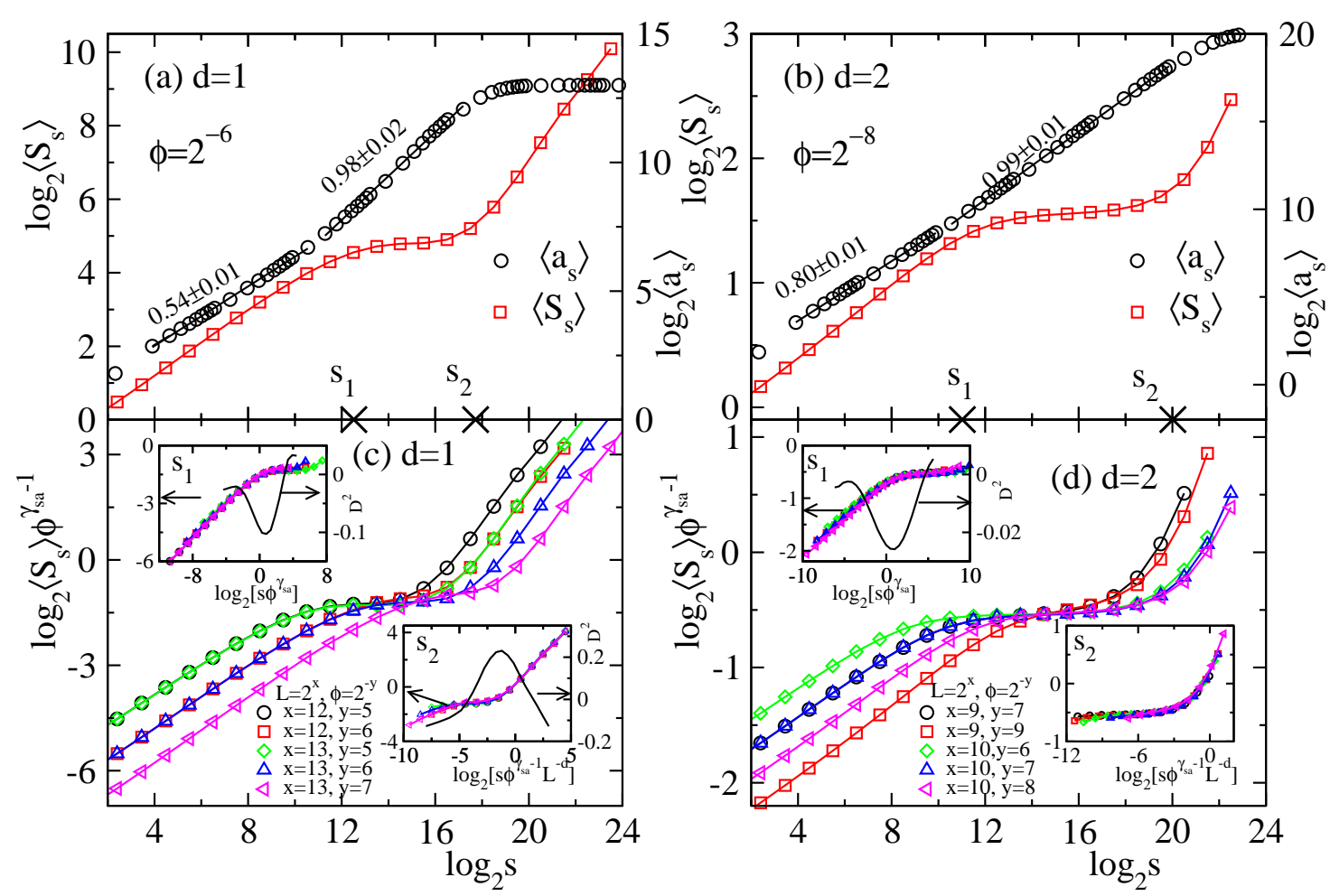

Figure 4: Plot of $\left\langle a_{s}\right\rangle$ against $s$ (black circle) and $\left\langle S_{s}\right\rangle$ against $s$ (red square) for $1 \mathrm{~d}$ in (a) and for $2 \mathrm{~d}$ in (b). Data for $1 \mathrm{~d}$ are collected for $\phi=2^{-6}$ and $L=8192$, whereas, that for $2 \mathrm{~d}$ are collected for $\phi=2^{-8}$ and $L=1024$. The rescaled $\left\langle S_{s}\right\rangle,\left\langle S_{s}\right\rangle \phi^{\gamma_{s a}-1}$ is plotted against $s$ for various values of $\phi$ and $L$ (see legend) in (c) for $1 \mathrm{~d}$ and in (d) for $2 \mathrm{~d}$. Insets: $\left\langle S_{s}\right\rangle \phi^{\gamma_{s a}-1}$ is plotted is plotted against scaled $s_{1}$ and $s_{2}$. Solid curve in each inset represents the double derivative of the scaled function against its argument.

which is then averaged over different avalanches of given size $s$ for a given $\phi$. The scaling of $\left\langle a_{s}\right\rangle$ and $\left\langle S_{s}\right\rangle$ with $s$ is given by

$$
\left\langle a_{s}\right\rangle \sim s^{\gamma_{a s}} \text { and }\left\langle S_{s}\right\rangle \sim s^{1-\gamma_{a s}}
$$

where $\gamma_{a s}$ is an exponent.

In the SWN regime, for a given system size $L$ and shortcut density $\phi$, the estimated values of $S_{s}$ and $\left\langle a_{s}\right\rangle$ are plotted against $s$ in Figs. 4(a) and 4(b) for 1d and 2d respectively. The values of $\gamma_{a s}$ are measured by linear least square fit through the data points of $\left\langle a_{s}\right\rangle$ for different region of $s$. For the avalanches of size $s<s_{1}$, it is found that $\gamma_{a s}=0.54 \pm 0.01$ in $1 \mathrm{~d}$ and $0.80 \pm 0.01$ in $2 \mathrm{~d}$ in close agreement with the known values of $\gamma_{a s}$ for SSM on RL as $\gamma_{a s}=1 / 2$ [33] in $1 \mathrm{~d}$ and $\gamma_{a s} \approx 0.78$ [34] in $2 \mathrm{~d}$. The scaling exponents of $\left\langle S_{s}\right\rangle$ are found as $\approx 0.5$ in $1 \mathrm{~d}$ and 0.22 in $2 \mathrm{~d}$ which is consistent with $1-\gamma_{a s}$. The scaling behaviour in this region is thus governed by the properties of the avalanches on $\mathrm{RL}(\phi=0)$. For the avalanches of size $s>s_{2}$, power law scaling of $\left\langle S_{s}\right\rangle$ and $\left\langle a_{s}\right\rangle$ are observed only for $1 \mathrm{~d}$ and no such scaling behaviour are observed for $2 \mathrm{~d}$. In $1 \mathrm{~d},\left\langle a_{s}\right\rangle$ saturates in this region though the avalanche size $s$ is increasing indicating $\gamma_{a s}=0$ and $1-\gamma_{a s}=1$. For the avalanches of 
intermediate sizes, $s_{1}<s<s_{2}, \gamma_{a s} \approx 1$, (0.98) in $1 \mathrm{~d}$ and (0.99) in $2 \mathrm{~d}$, as shown in Figs. 4(a) and 4(b), respectively. Accordingly, $\left\langle S_{s}\right\rangle$ remains constant against $s$. Since $\gamma_{a s}=1$ on $\mathrm{RN}$ in both $1 \mathrm{~d}$ and $2 \mathrm{~d}$ [16], the scaling behaviour in this region is then governed by the properties of the avalanches on random network. The values of $s_{1}$ and $s_{2}$ can be obtained determining the points of inflections in the plots of $\left\langle S_{s}\right\rangle$ against $s$. These plots for different $\phi$ and $L$ are found to be shifted both vertically and horizontally. A scaling form for $\left\langle S_{s}\right\rangle$ as well as for the crossover sizes $s_{1}$ and $s_{2}$ can be established considering the fact that there exits a characteristic length $\xi \sim \phi^{-1 / d}$ where $d$ is the dimensionality of the lattice, below which SWN belongs to "large world", the RL regime and beyond which it behaves as "smallworld", the RN regime [36, 37, 38]. At this length scale, the avalanche area $a \approx \xi^{d} \sim 1 / \phi$ and $\left\langle S_{s}\right\rangle$ can accordingly be obtained as

$$
\left\langle S_{s}\right\rangle=\frac{s}{a} \sim \phi^{1-\gamma_{s a}}
$$

where $\gamma_{s a}=1 / \gamma_{a s}$. In Figs. 4(c) and 4(d), the scaled average height $\left\langle S_{s}\right\rangle \phi^{\gamma_{s a}-1}$ are plotted against $s$ for $1 \mathrm{~d}$ and $2 \mathrm{~d}$ respectively for different values of $L$ and $\phi$. It can be seen that in the intermediate range of $s$, the plots in both the figures have a common constant height independent of $\phi$ and $L$. The avalanches confined within $\xi$ are expected to have $s<s_{1}$ whereas the avalanches extend beyond $\xi$ and comparable to $L$ are expected to have $s>s_{2}$. Hence, two crossover sizes $s_{1}$ and $s_{2}$ correspond to two length scales, $\xi$ and $L$, present in this system. The scaling form of $s_{1}$ and $s_{2}$, the crossover avalanche sizes, are then given by

$$
s_{1}=\bar{S} \xi^{d} \sim \phi^{-\gamma_{s a}} \quad \text { and } \quad s_{2}=\bar{S} L^{d} \sim \phi^{1-\gamma_{s a}} L^{d}
$$

where $\bar{S}$ is the value of $\left\langle S_{s}\right\rangle$ in the intermediate region. Each data set of $\left\langle S_{s}\right\rangle$ is subdivided into two sets breaking it at the middle of the intermediate region. The scaling forms of $s_{1}$ and $s_{2}$ are verified independently by data collapse for $\left\langle S_{s}\right\rangle$ in different regions as shown in the insets of Figs. 4(c) and 4(d). Identifying the point of inflection in the collapsed plots (taking double derivative $D^{2}$ with respect to the argument), the values of $s_{1}$ and $s_{2}$ can be estimated. The positions of the dips and peaks of $D^{2}$ corresponding to the values of $s_{1}$ and $s_{2}$ are shown in the respective insets of Figs. 4(c) and 4(d). However, the positions of the dips and peaks are found slightly shifted from the origin in the logarithmic scale due to associated metric factors in $s_{1}$ and $s_{2}$ (Eq. 8). Estimating the corresponding matric factors, the values of $s_{1}$ and $s_{2}$ in $1 \mathrm{~d}$ for $L=2^{13}$ and $\phi=2^{-6}$ are obtained as: $s_{1} \approx 2^{12.5}$ and $s_{2} \approx 2^{17.7}$. In $2 \mathrm{~d}$, however, no inflection point is found for $s_{2}$. Therefore, for $L=2^{10}$ and $\phi=2^{-8}$, the value of $s_{1}$ is obtained incorporating the metric factor as $s_{1}=2^{10.97}$ and that of $s_{2}$ by direct estimation from Eq. (8) as $s_{2}=2^{20}$. The values of $s_{1}$ and $s_{2}$ are marked by crosses in Figs. 4(a) and 4(b). It has been verified that $\left\langle S_{s}\right\rangle$ of deterministic BTW-type sandpile also exhibits similar scaling behaviour at different regions of $s$ in the SWN regime.

The ensemble of avalanches that appear in the steady states of these systems then can be classified into three different categories. The avalanches with size $s<s_{1}$ are mostly fragment less and confined on the large world or RL. The growth of their average height follow different power law scaling with the avalanche size $s$ in $1 \mathrm{~d}$ and $2 \mathrm{~d}$. In the intermediate 

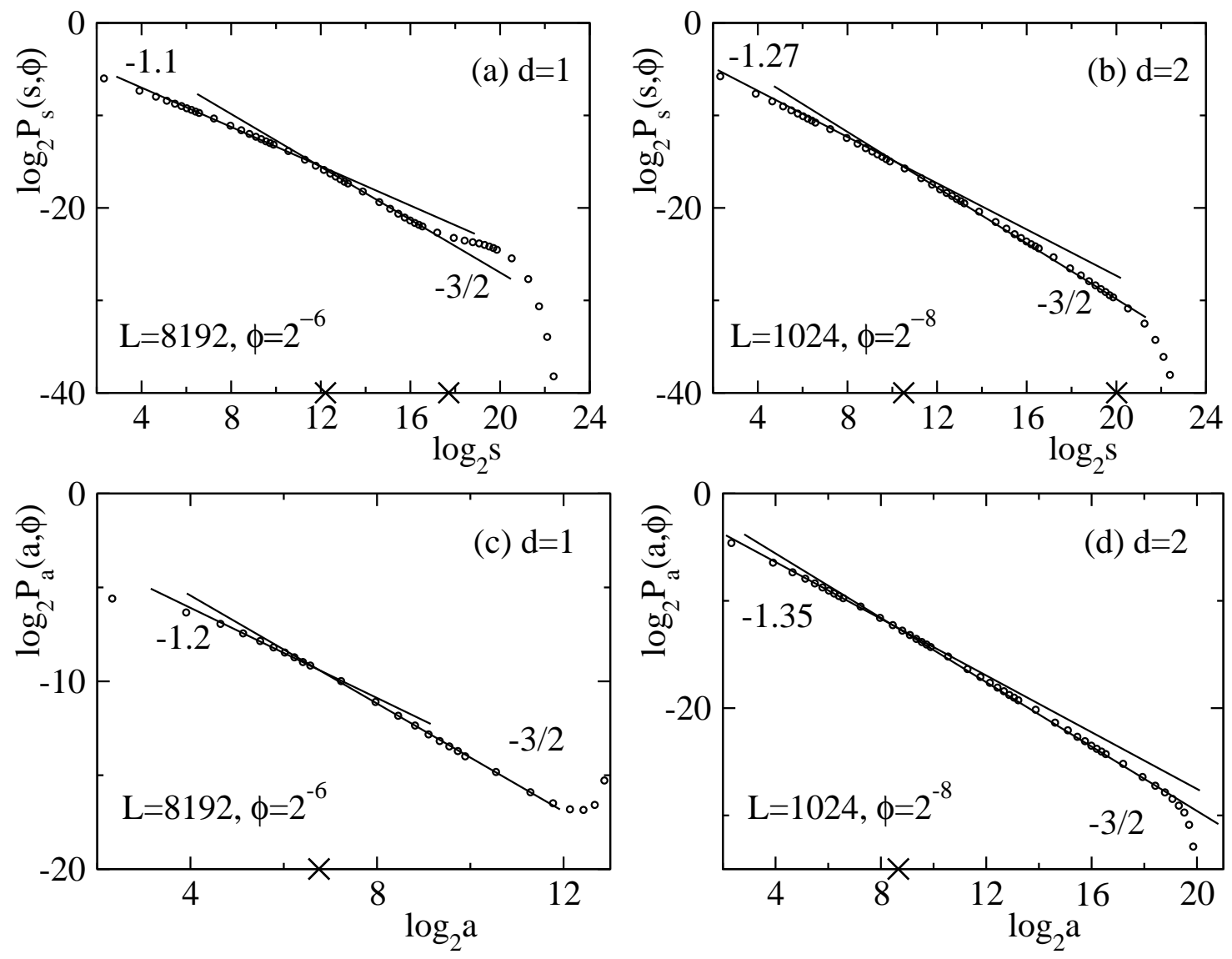

Figure 5: Plot of $P_{s}(s, \phi)$ against $s$ in (a) for $1 \mathrm{~d}$ with $L=8192$ for $\phi=2^{-6}$ and in (b) for $2 \mathrm{~d}, L=1024$ for $\phi=2^{-8}$. Plot of $P_{a}(a, \phi)$ against $a$ in (c) for $1 \mathrm{~d}$ and (d) for $2 \mathrm{~d}$ with the same $L$ and $\phi$ as in (a) and (b) respectively. The solid lines with required slope (as indicated beside) represent two distinct scaling behaviour in two regimes.

region $s_{1}<s<s_{2}$, the avalanche clusters are sparse, different parts of the same cluster are connected by the links of the network. These clusters are then appearing on the smallworld or on the network. Their average height do not grow with their avalanche size as the avalanche cluster become sparse both in $1 \mathrm{~d}$ and $2 \mathrm{~d}$. As the size of the avalanches exceed $s_{2}$, the avalanche cluster properties are very different in one and two dimensions. In this region, the avalanche clusters become a compact over grown single cluster in $1 \mathrm{~d}$ whereas in $2 \mathrm{~d}$ they grow in size but remain sparse.

\subsection{Avalanche size and area distributions}

The exponents associated with the power-law scaling of the probability distributions of avalanche property $x \in\{s, a\}$ are generally use to characterize the critical steady state of sandpile model. For a given $\phi$, the probability to have an avalanche of property $x$ is given by $P_{x}(x, \phi)=N_{x, \phi} / N_{\text {tot }}$ where $N_{x, \phi}$ is the number of avalanches having property $x$ out of total number of avalanches $N_{\text {tot }}$ generated at the steady state. Since the avalanches occurring on a given SWN (of particular $\phi$ ) exhibits different geometrical properties according to 
their size $s$, the avalanche size distribution $P_{s}(s, \phi)$ should exhibit power-law scaling with different exponents in different regimes in a single distribution. A similar scaling behaviour of avalanche area distribution $P_{a}(s, \phi)$ is also expected, because for each crossover size there should a associated crossover area. A generalized scaling form of $P_{x}(x, \phi)$ among any two regimes is proposed as

$$
P_{x}(x, \phi)= \begin{cases}x^{-\tau_{x 1}} \mathrm{f}_{\mathbf{\times}}\left(\frac{x}{x_{c}(\phi)}\right) & \text { for } x \leqslant x_{c} \\ x^{-\tau_{x 2}} \mathrm{~g}_{\times}\left(\frac{x}{x_{c}(\phi)}\right) & \text { for } x \geqslant x_{c}\end{cases}
$$

where $x_{c}$ is the crossover value of the property $x, \mathrm{f}_{\mathrm{x}}$ and $\mathrm{g}_{\mathrm{x}}$ are the respective scaling functions and $\tau_{x 1}, \tau_{x 2}$ are the corresponding critical exponents in the respective region. $P_{x}(x, \phi)$ are estimated on a $1 \mathrm{~d}$ lattice of size $L=8192$ for $\phi=2^{-6}$, and on a $2 \mathrm{~d}$ square lattice of size $L=1024$ for $\phi=2^{-8}$. The distributions of avalanche size $P_{s}(s, \phi)$ are presented in Figs. 5(a) and 5(b) respectively for $1 \mathrm{~d}$ and $2 \mathrm{~d}$ lattices. It can be seen that the distributions $P_{s}(s, \phi)$ in Figs. 5(a) and 5(b) do not follow a single power law scaling over the whole range of avalanche sizes. There are three distinct regions of avalanche size $s$, separated by two crossover sizes $s_{1}$ and $s_{2}$, which were observed in case of geometrical aspect of the avalanches, indicated by crosses. $P_{s}(s, \phi)$ seems to have different scaling exponents in different regions of $s$. It could be observed that the avalanches of size $s>s_{2}$ are large avalanches and do not contribute to the power-law scaling. Hence, the crossover at $s_{1}$ and the associated scaling properties will be analyzed in the following. Since the avalanches in the region $s<s_{1}$ correspond to the avalanches on RL, the scaling behaviour of $P_{s}(s, \phi)$ should either be that of Manna scaling. Whereas, for the region $s>s_{1}$ the avalanches correspond to those on the network, and hence the scaling behaviour should follow MF scaling on network. The avalanche size distribution exponent $\tau_{s}$ of SSM on a regular 1d lattice is known to be 1.1 [39, 33, 40, 41, 29] and that on a regular $2 \mathrm{~d}$ lattice is $\approx 1.28$ [15, 29]. Whereas the MF value of $\tau_{s}$ is $3 / 2$ on both $1 \mathrm{~d}$ and $2 \mathrm{~d}$. Two straight lines of respective slopes are plotted in Figs. 5(a) and 5(b) as guide to the eye. It can be noticed that a reasonable portion of data points of $P_{s}(s, \phi)$ obtained for SWN do follow the respective scaling forms in both the dimensions. The avalanche area distributions $P_{a}(a, \phi)$ are presented in Figs. 5(c) and 5(d) respectively for $1 \mathrm{~d}$ and $2 \mathrm{~d}$ lattices. A similar behaviour as that of $P_{s}(s, \phi)$ is observed for $P_{a}(a, \phi)$ too. The value of crossover area $a_{1}$ associated with crossover size $s_{1}$ can be estimated from the conditional expectation relation $a_{1} \sim s_{1}^{\gamma_{a s}}$. Taking the respective values of $s_{1}$ and $\gamma_{a s}$, the values of $a_{1}$ are estimated as $2^{6.75}$ for $1 \mathrm{~d}$ and $2^{8.77}$ for $2 \mathrm{~d}$ and are indicated by crosses in Figs. 5(c) and 5. (d) respectively. The avalanche area distribution exponent $\tau_{a}$ of SSM on a regular 1d lattice is known to be 1.2 and that on a regular $2 \mathrm{~d}$ lattice is $\approx 1.35$ [24], whereas the MF value is $\tau_{a}=3 / 2$. It can be seen that data points of $P_{a}(a, \phi)$ follow SSM scaling in $a<a_{1}$ regime and MF scaling in $a>a_{1}$ regime in both the dimensions.

It has already been known that the distribution functions of stochastic sandpile model follow FSS both on $\operatorname{RL}(\phi=0)$ as well as on $\operatorname{RN}(\phi=1)$ [14, 28]. It is then important to verify whether the probability distributions of DSSM follow FSS or not in the SWN regime. 

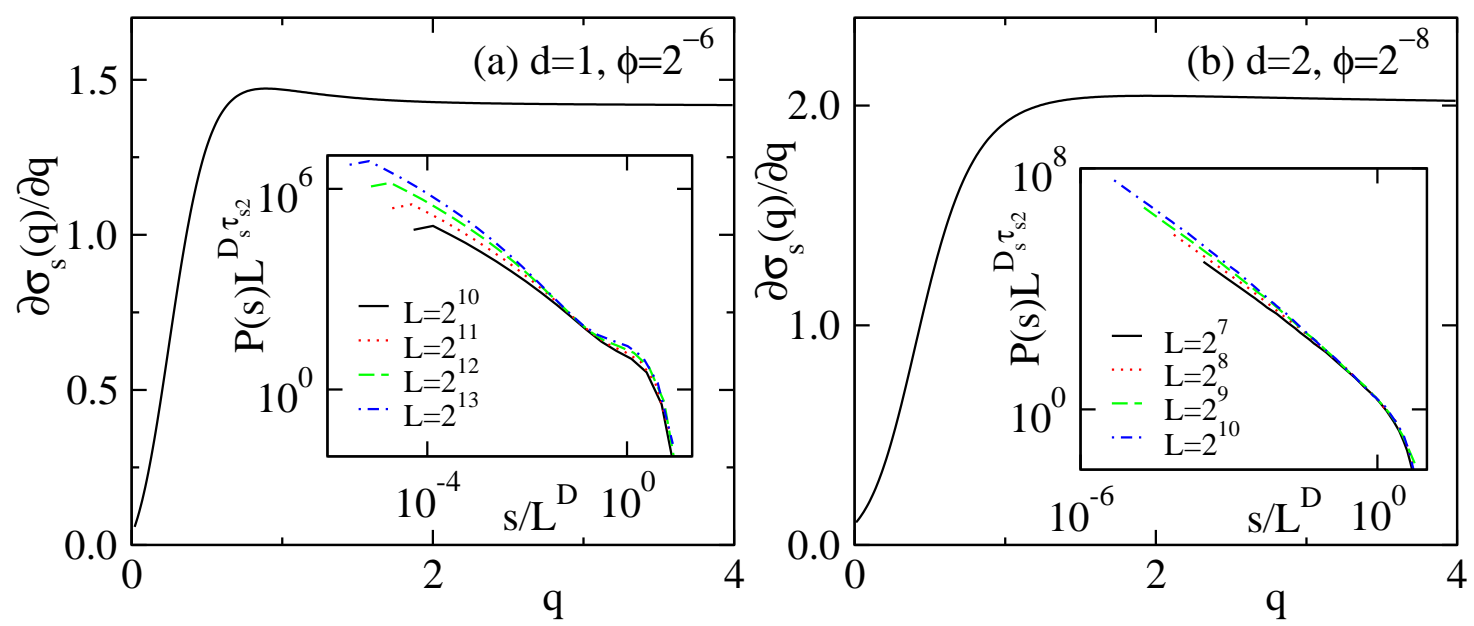

Figure 6: Plot of $\partial \sigma_{s}(q, \phi) / \partial q$ against $q$ in (a) for $1 \mathrm{~d}$ with $\phi=2^{-6}$ and in (b) for $2 \mathrm{~d}$ with $\phi=2^{-8}$. Inset: Attempt to collapse the data of avalanche size distribution for different system sizes taking $\tau_{s}=3 / 2$, and respective values of $D_{s}$.

The FSS scaling form of size distribution function for a given fixed $\phi$ is assumed as

$$
P(s, L) \approx s^{-\tau} f\left(s / L^{D_{s}}\right)
$$

where $D_{s}$ is the capacity dimension. Moment analysis of the avalanche size has been performed for both the dimensions. The average $q$ th moment of avalanche size $s$ for a given $\phi$ is defined as

$$
\left\langle s^{q}\right\rangle=\int_{0}^{s_{\max }} s^{q} P(s, L) d s \sim L^{\sigma_{s}(q)}
$$

where $\sigma_{s}(q)=\left[q+1-\tau_{s}\right] D_{s}$ is the moment scaling function. The values of $\sigma_{s}(q)$ for different values of $\phi$ are obtained and its derivatives with respect to $q$ are plotted against $q$ in Fig. 6 (a) for $1 \mathrm{~d}$ with $\phi=2^{-6}$ and in Fig. 6 (b) for $2 \mathrm{~d}$ with $\phi=2^{-8}$. It can be seen that $\partial \sigma_{s}(q) / \partial q$ converges for large $q$ in both the cases and the values of capacity dimension estimated as $D_{s} \approx 1.42$ and $\approx 2$ for $1 \mathrm{~d}$ and $2 \mathrm{~d}$, respectively. It should be noted here that for the given values of $\phi$, the distribution functions consist of two scaling forms with exponents $\tau_{s 1}$ and $\tau_{s 2}$. An attempt has been made to collapse the data by plotting the scaled distribution $P(s, \phi) L^{D_{s} \tau_{s 2}}$ against the scaled variable $s / L^{D_{s}}$ in the respective insets. For both the dimensions, $\tau_{s 2}$ is taken as $3 / 2$. Clearly, the collapse does not work for the whole range of $s$. The deviation is more prominent in the small avalanche size region where the avalanches follow usual stochastic scaling of the respective dimensions. It is then important to note that in the SWN regime FSS of the probability distributions can not be verified for the stochastic sandpile model too because multi-scaling feature of the same exists. A similar observation is also reported by Benella et.al. [42] in a recent study of avalanching systems with long range connectivity. Since FSS fails in the SWN regime, the $\phi$ dependent coexistence scaling could be useful to verify the scaling form of the probability distribution functions. 

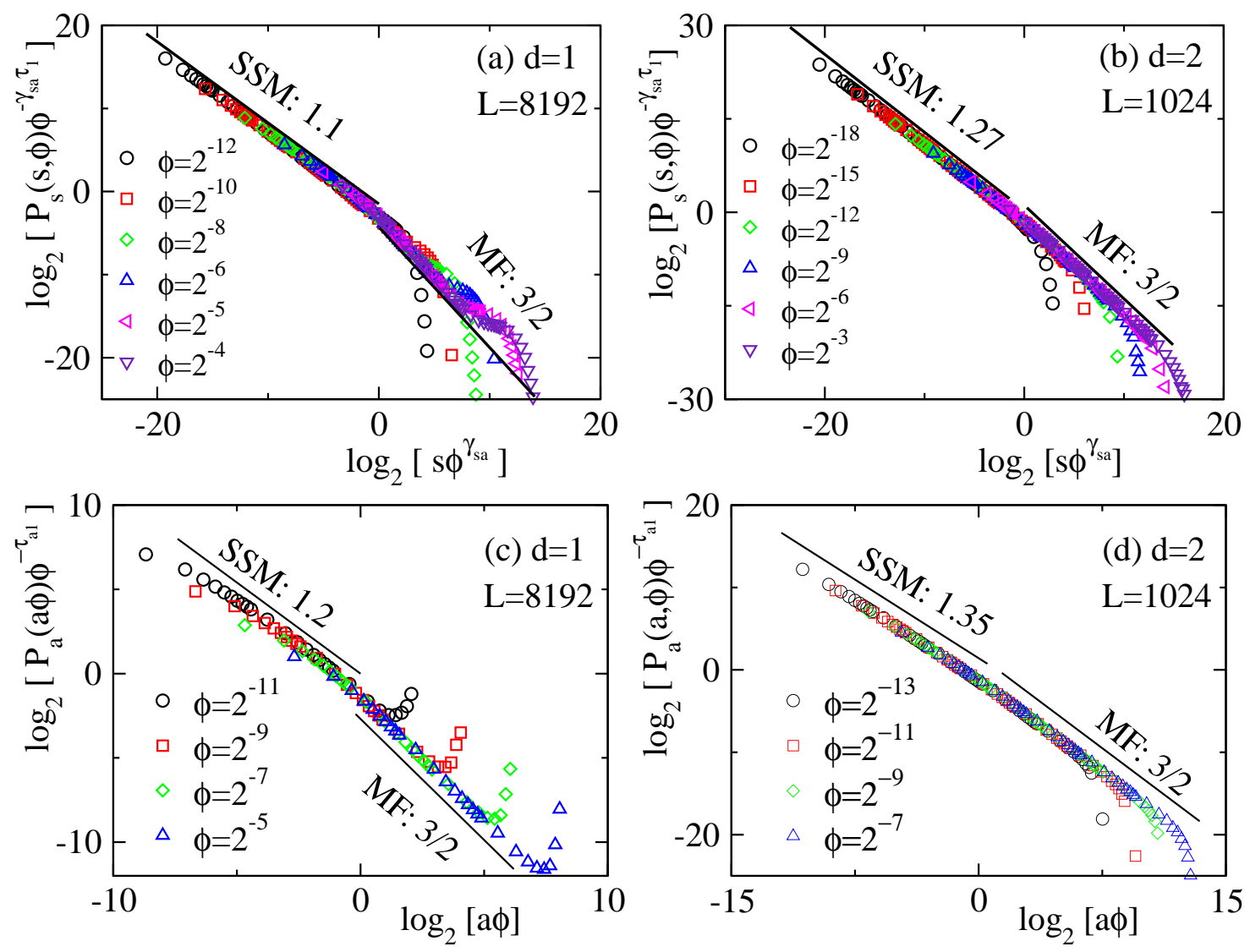

Figure 7: Plot of scaled size distributions against scaled variable for selected values of $\phi$ in (a) for $1 \mathrm{~d}$ and in (b) for $2 \mathrm{~d}$. The scaled area distributions for several values of $\phi$ are shown in (c) for $1 \mathrm{~d}$ and in (d) for $2 \mathrm{~d}$. The system size considered for $1 \mathrm{~d}$ is $L=8192$, and for $2 \mathrm{~d}$ is $L=1024$

\subsection{Coexistence scaling}

Verification of the scaling form given in Eq. (9) can be performed for the two regions taking crossover value $x_{c}=x_{1}$. At $x_{1}(\phi)$ the values of $P_{x}(x, \phi)$ are same for both the regions. Since $s_{1} \sim \phi^{-\gamma_{s a}}$, then one should have $\mathrm{f}_{\mathrm{s}}(1)=\phi^{-\left(\tau_{s 1}-\tau_{s 2}\right) \gamma_{s a}} \mathrm{~g}_{\mathrm{s}}(1)$. Following Ref. [19], the scaled size distribution can be obtained as

$$
P_{s}(s, \phi) \phi^{-\gamma_{s a} \tau_{s 1}}= \begin{cases}\left(s \phi^{\gamma_{s a}}\right)^{-\tau_{s 1}} \mathrm{f}_{\mathrm{s}}\left(s \phi^{\gamma_{s a}}\right) & \text { for } s \leqslant s_{1} \\ \left(s \phi^{\gamma_{s a}}\right)^{-\tau_{s 2}} \mathrm{f}_{\mathbf{s}}\left(s \phi^{\gamma_{s a}}\right) & \text { for } s \geqslant s_{1}\end{cases}
$$

Similarly considering $a_{1} \sim s_{1}^{\gamma_{a s}} \sim \phi^{-1}$ a scaled area distribution can be written as

$$
P_{a}(a, \phi) \phi^{-\tau_{a 1}}= \begin{cases}(a \phi)^{-\tau_{a 1}} \mathrm{f}_{\mathrm{a}}(a \phi) & \text { for } a \leqslant a_{1} \\ (a \phi)^{-\tau_{a 2}} \mathrm{f}_{\mathrm{a}}(a \phi) & \text { for } a \geqslant a_{1}\end{cases}
$$

To verify the scaling forms given in Eqs. (12) and (13), the scaled distributions are plotted in Fig. 7 against their respective scaled variable. Data of size distributions are presented in 

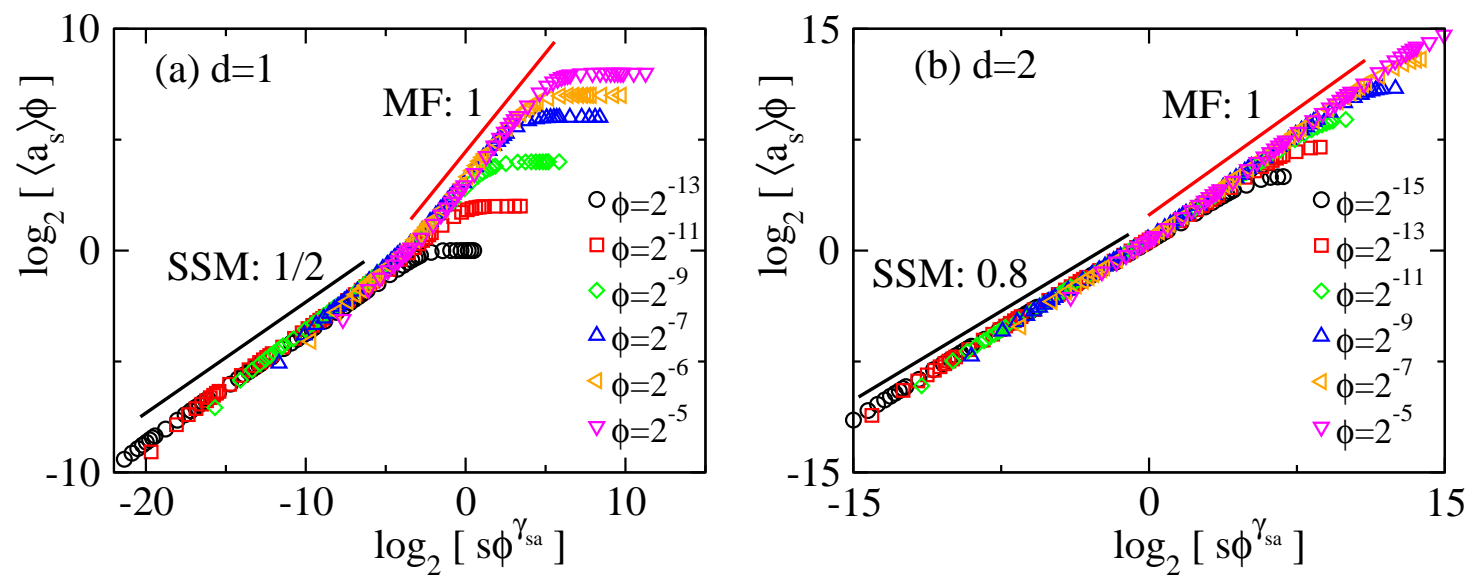

Figure 8: Plot of scaled average area against scaled variable for selected values of $\phi$ in (a) for $1 \mathrm{~d}$ with $L=8192$ and (b) for 2 d with $L=1024$. Reasonable data collapse verify the scaling forms given in Eq. (14). Straight lines with with required slope are guide to the eyes.

Figs. 7(a) and 7(b) for $1 \mathrm{~d}$ and $2 \mathrm{~d}$ respectively. It can be seen that a good data collapse is obtained using $\gamma_{s a}=2, \tau_{s 1}=1.11$ for $1 \mathrm{~d}$ and using $\gamma_{s a}=1.26, \tau_{s 1}=1.27$ for $2 \mathrm{~d}$. Similarly Figs. 7 (c) and 7 (d) represent the data of scaled area distributions for $1 \mathrm{~d}$ and $2 \mathrm{~d}$ respectively. A reasonable collapse are also observed taking $\tau_{a 1}=1.2$ for $1 \mathrm{~d}$ and $\tau_{a 1}=1.35$ for $2 \mathrm{~d}$. The straight lines with required slopes in the respective regions are guide to the eye. It confirms the validity of the proposed scaling function forms given in Eq. (91).

The coexistence scaling of the average height $\left\langle S_{s}\right\rangle$ around crossover size $s_{1}$ and $s_{2}$ has already been demonstrated in previous section (Figs. 4(c) and 4(d)). Another geometrical property of toppling surface, the average area $\left\langle a_{s}\right\rangle$ of a given size $s$ also exhibits multiple scaling forms in different regions of $s$. A coexistence scaling form of $\left\langle a_{s}\right\rangle$ around the crossover size $s_{1}$ can be written as

$$
\left\langle a_{s}\right\rangle= \begin{cases}s^{\gamma_{a s 1}} f_{a s}\left(\frac{s}{s_{1}}\right) & \text { for } s \leqslant s_{1} \\ s^{\gamma_{a s 2}} g_{a s}\left(\frac{s}{s_{1}}\right) & \text { for } s \geqslant s_{1}\end{cases}
$$

where, $f_{a s}$ and $g_{a s}$ are the scaling functions in respective regions and $\gamma_{a s 1}$ and $\gamma_{a s 2}$ are the exponents in the regime $s \leqslant s_{1}$ and $s \geqslant s_{1}$ respectively. Note that at $s=s_{1}$ the value of $\left\langle a_{s}\right\rangle$ will be same for both the regions and hence $f_{a s}(1)=s_{1}^{\gamma_{a s 2}-\gamma_{a s 1}} g_{a s}(1)$. Considering $\gamma_{s a}=1 / \gamma_{a s}$, Eq. (14) can be written in terms of single scaling function as

$$
\left\langle a_{s}\right\rangle \phi= \begin{cases}\left(s \phi^{\gamma_{s a 1}}\right)^{\gamma_{a s 1}} f_{a s}\left(s \phi^{\gamma_{s a 1}}\right) & \text { for } s \leqslant s_{1} \\ \left(s \phi^{\gamma_{s a 1}}\right)^{\gamma_{a s 2}} f_{a s}\left(s \phi^{\gamma_{s a 1}}\right) & \text { for } s \geqslant s_{1}\end{cases}
$$

A plot of $\left\langle a_{s}\right\rangle \phi$ against $s \phi^{\gamma_{s a}}$ for different values of $\phi$ should fall on a single curve. Satisfactory collapse of date are observed as shown in Fig. 8(a) for 1d and in Fig. 8(b) for 2d. Moreover, the scaling function represents two different scaling behaviour with two different exponents, 

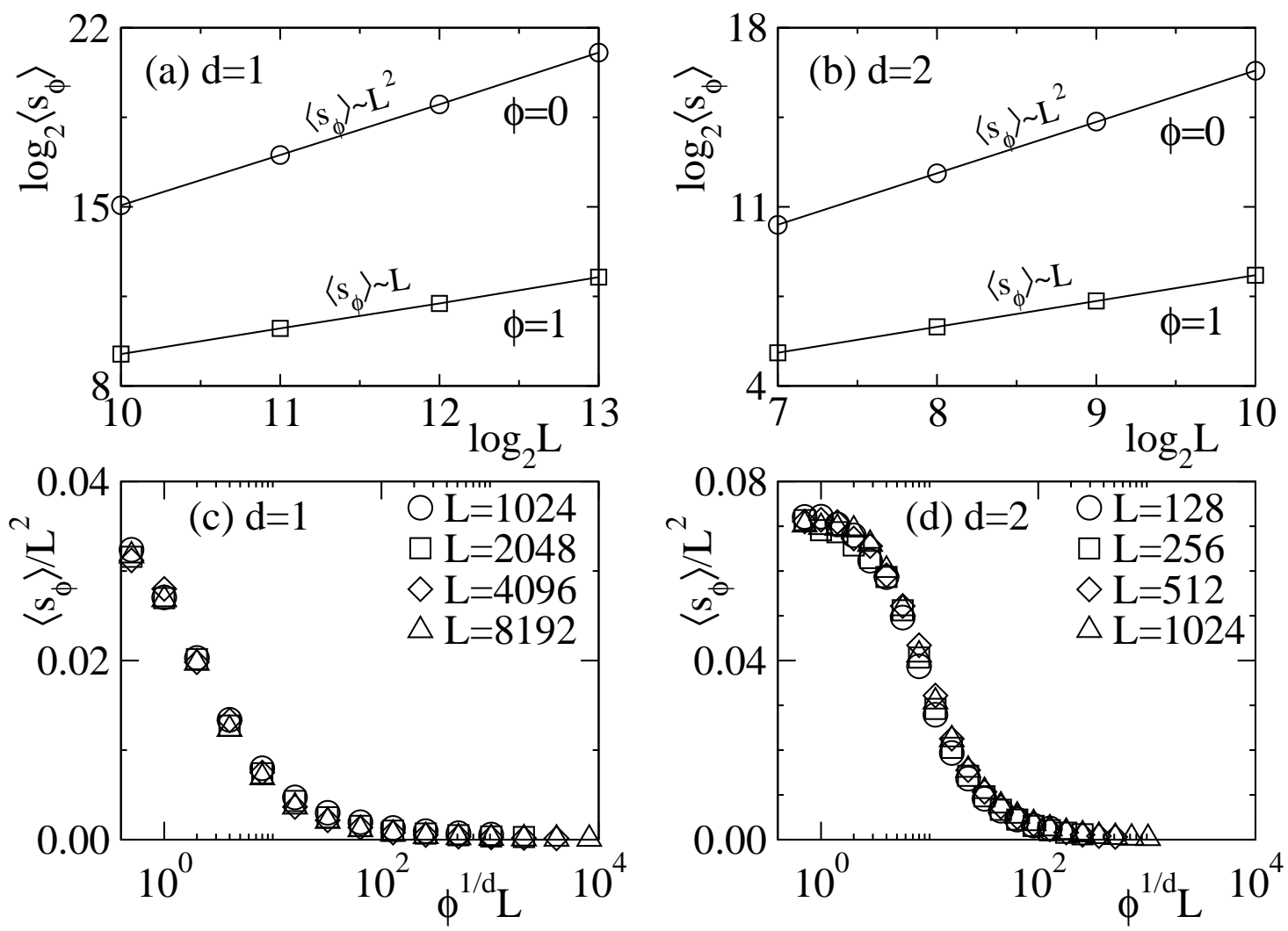

Figure 9: Plot of $\left\langle s_{\phi}\right\rangle$ against $L$ for $\phi=0(\bigcirc)$ and $\phi=1$ ( $\square$ ) in (a) for 1d and in (b) for 2d. Plot of scaled average size $\left\langle s_{\phi}\right\rangle / L^{2}$ against scaled variable $\phi^{1 / d} L$ for (c) $d=1$ and (d) $d=2$. Different symbols correspond to different system sizes $L$.

$1 / 2$ and 1 in $1 \mathrm{~d}$ and 0.8 and 1 in $2 \mathrm{~d}$, as indicated by straight lines with the respective slopes.

It is then important to notice that if a dynamical model like sandpile is studied on SWN, multiple scaling forms of an event size will coexist in the distribution of the event sizes. In other words, SWN can be considered as a segregator of several scaling forms that appear in the event size distribution.

\subsection{Sand transport: diffusivity and scaling}

It is known in the literature that, the critical properties the stochastic model on RL $(\phi=0)$ governed by the diffusive nature of the sand transport [33, 24], i.e., $r \sim \sqrt{t}$. However, as the SWN evolves to RN $(\phi=1)$, it is expected that the diffusive nature changes to superdiffusive one i.e., $r \sim t$, as it is already observed for the deterministic model [19]. In order to verify the same, the average avalanche size (time to diffuse) on a given system size $L$ (length to diffuse) for a given $\phi$ is defined as

$$
\left\langle s_{\phi}\right\rangle=\int s P_{s}(s, \phi) d s
$$


At the extreme values of $\phi,\left\langle s_{\phi}\right\rangle$ is plotted against system size $L$ in Figs. 9(a) and 9(b) for $1 \mathrm{~d}$ and $2 \mathrm{~d}$, respectively. It can be seen that in both the dimensions, the diffusive behaviour of the model, $\left\langle s_{\phi}\right\rangle \sim L^{2}$ holds for $\phi=0$, whereas the behaviour changes to super-diffusive for other extreme value of $\phi$ i.e., $\left\langle s_{\phi}\right\rangle \sim L$ for $\phi=1$. It is now important to check the scaling behaviour of $\left\langle s_{\phi}\right\rangle$ with $\phi$ on SWNs. A generalized scaling form of $\left\langle s_{\phi}\right\rangle$ can be written as

$$
\left\langle s_{\phi}\right\rangle=L^{2} \mathcal{G}\left(\phi^{1 / d} L\right)
$$

where $d$ is the space dimension. The behaviour of the scaling function $\mathcal{G}(x)$ is given by,

$$
\mathcal{G}(x) \propto \begin{cases}\text { constant, } & x \ll 1 \\ 1 / x, & x \gg 1\end{cases}
$$

In order to verify the above scaling form, $\left\langle s_{\phi}\right\rangle$ is calculated for different system sizes over the full range of $\phi$ for both $1 \mathrm{~d}$ and $2 \mathrm{~d}$. The scaled average size $\left\langle s_{\phi}\right\rangle / L^{2}$ is plotted against the scaled variable $\phi^{1 / d} L$ in Figs. 9(c) and 9(d) for $1 \mathrm{~d}$ and $2 \mathrm{~d}$ respectively. Taking the respective $d$ values, reasonable collapse of data are observed in both the cases. Note that, for $2 \mathrm{~d}$, the limiting values of $\left\langle s_{\phi}\right\rangle / L^{2}$ as $\phi \rightarrow 0$ is found to be 0.7 which is consistence with the relations $2\left\langle s_{\phi}\right\rangle=\left\langle n_{\phi}\right\rangle$ and $\left\langle n_{\phi}\right\rangle \approx 0.14 L^{2}$, for $L \rightarrow \infty$ [43]. However, for $1 \mathrm{~d},\left\langle s_{\phi}\right\rangle / L^{2}$ is found to be $\approx 0.031$ as $\phi \rightarrow 0$.

\section{Summary and Conclusion}

A dissipative stochastic sandpile model (DSSM) is constructed and studied on SWN both in $1 \mathrm{~d}$ and $2 \mathrm{~d}$ varying the shortcut density $\phi$. As $\phi$ varies from 0 to 1 , RL evolves to RN via a series of SWNs. Since the critical behaviour of the stochastic model on RL as well as on RN is known, emphasis is given in analyzing the critical properties of the model on SWN regime $\left(2^{-12}<\phi<0.1\right)$. Several new geometrical quantities such as toppling surface and its fragmentation, compactness and fluctuation in the fragment size are defined and characterized as a function of avalanche size $s$ for a given $\phi$. The average height $\left\langle S_{s}\right\rangle$ and average area $\left\langle a_{s}\right\rangle$ of the toppling surface are found to have three regimes of avalanche size $s$, separated by two crossover sizes $s_{1}$ and $s_{2}\left(s_{1}<s_{2}\right)$. Below $s_{1}$, the avalanches are found to be compact as that are on RL. For $s_{1}<s<s_{2}$ regime, the avalanches are fragmented in many sub-clusters that are connected by long-ranged link of the network. Novel scaling forms of $s_{1}$ and $s_{2}$ as well as that of $\left\langle S_{s}\right\rangle$ are developed and numerically verified. Distributions of avalanche size $s$ and area $a$ are also found to exhibit two scaling forms about the crossover size $s_{1}$. Below $s_{1}$ it is Manna scaling on RL whereas above $s_{1}$ it is MF scaling on RN. Since two scaling forms appear simultaneously in the SWN regime, the probability distributions of various avalanche properties of DSSM do not follow the usual FSS in contrary to the fact that stochastic sandpile model follow FSS on RL. Around the crossover size a coexistence scaling form of the distributions and the expectation value are developed and numerically verified. The sand transport behaviour in DSSM is found to change from diffusive to super diffusive nature as SWN evolve from RL to RN. A generalized scaling form of diffusivity is identified that satisfactorily explains such crossover behaviour. 
Acknowledgments: This work is partially supported by DST, Government of India through project No. SR/S2/CMP-61/2008. Availability of computational facility, "Newton HPC" under DST-FIST project (No. SR/FST/PSII-020/2009) Government of India, of Department of Physics, IIT Guwahati is gratefully acknowledged.

\section{References}

[1] P. Bak, How Nature Works: The Science of Self-Organized Criticality, Copernicus, New York, 1996.

[2] H. J. Jensen, Self-Organized Criticality, Cambridge University Press, Cambridge, 1998.

[3] G. Pruessner, Self- Organized Criticality: Theory, Models and Characterization, Cambridge University Press, Cambridge, 2012.

[4] L. de Arcangelis, C. Perrone-Capano, and H. J. Herrmann, Phys. Rev. Lett. 96, 028107 (2006).

[5] J. Hesse and T. Gross, Frontiers in Systems Neuroscience 8, 166 (2014).

[6] S. Lise and M. Paczuski, Phys. Rev. Lett. 88, 228301 (2002).

[7] D. Hughes, M. Paczuski, R. O. Dendy, P. Helander, and K. G. McClements, Phys. Rev. Lett. 90, 131101 (2003).

[8] A. E. Motter and Y.-C. Lai, Phys. Rev. E 66, 065102 (2002).

[9] B. Carreras, D. Newman, I. Dobson, and A. Poole, Circuits and Systems I: Regular Papers, IEEE Transactions on 51, 1733 (2004).

[10] D. Watts and S. Strogatz, Nature 393, 440 (1998).

[11] P. Bak, C. Tang, and K. Wiesenfeld, Phys. Rev. Lett. 59, 381 (1987).

[12] P. Bak, C. Tang, and K. Wiesenfeld, Phys. Rev. A 38, 364 (1988).

[13] M. DeMenech, A. L. Stella, and C. Tebaldi, Phys. Rev. E 58, R2677 (1998).

[14] C. Tebaldi, M. DeMenech, and A. L. Stella, Phys. Rev. Lett. 83, 3952 (1999).

[15] S. Lübeck, Phys. Rev. E 61, 204 (2000).

[16] K. Christensen and Z. Olami, Phys. Rev. E 48, 3361 (1993).

[17] E. Bonabeau, Journal of the Physical Society of Japan 64, 327 (1995).

[18] K.-I. Goh, D.-S. Lee, B. Kahng, and D. Kim, Phys. Rev. Lett. 91, 148701 (2003).

[19] H. Bhaumik and S. B. Santra, Phys. Rev. E 88, 062817 (2013).

[20] S. S. Manna, J. Phys. A 24, L363 (1991).

[21] D. Dhar, Physica A 263, 4 (1999).

[22] V. Frette, K. Christensen, A. Malthe-Sørenssen, J. Feder, T. Jøssang, and P. Meakin, Nature 379, 49 (1996).

[23] H. N. Huynh, L. Y. Chew, and G. Pruessner, Phys. Rev. E 82, 042103 (2010).

[24] H. Huynh, G. Pruessner, and L. Chew, J. Stat. Mech 2011, P09024 (2011).

[25] H. N. Huynh and G. Pruessner, Phys. Rev. E 85, 061133 (2012).

[26] M. E. J. Newman, A.-L. Barabási, and D. J. Watts, The Structure and Dynamics of Networks: (Princeton Studies in Complexity), Princeton University Press, Princeton, NJ, USA, 2006.

[27] O. Malcai, Y. Shilo, and O. Biham, Phys. Rev. E 73, 056125 (2006).

[28] S. A. Moosavi and A. Montakhab, Phys. Rev. E 89, 052139 (2014).

[29] H. Bhaumik and S. B. Santra, Phys. Rev. E 94, 062138 (2016).

[30] J. A. Ahmed and S. B. Santra, Europhys. Lett. 90, 50006 (2010).

[31] J. A. Ahmed and S. Santra, Physica A 391, 5332 (2012).

[32] H. Bhaumik, J. A. Ahmed, and S. B. Santra, Phys. Rev. E 90, 062136 (2014).

[33] H. Nakanishi and K. Sneppen, Phys. Rev. E 55, 4012 (1997).

[34] A. Ben-Hur and O. Biham, Phys. Rev. E 53, R1317 (1996).

[35] S. B. Santra, S. R. Chanu, and D. Deb, Phys. Rev. E 75, 041122 (2007).

[36] M. E. J. Newman and D. J. Watts, Phys. Rev. E 60, 7332 (1999).

[37] M. E. J. Newman and D. J. Watts, Physics Letters A 263, 341 (1999).

[38] M. A. de Mendes, C. F. Moukarzel, and T. J. P. Penna, Europhys. Lett. 50, 574 (2000).

[39] R. Dickman and J. M. M. Campelo, Phys. Rev. E 67, 066111 (2003). 
[40] R. Dickman, M. A. Muñoz, A. Vespignani, and S. Zapperi, Brazilian Journal of Physics 30, 27 (2000). [41] J. A. Bonachela and M. A. Muñoz, Phys. Rev. E 78, 041102 (2008).

[42] S. Benella, G. Consolini, F. Giannattasio, T. T. Chang, and M. Echim, Entropy 19, 383 (2017).

[43] Y. Shilo and O. Biham, Phys. Rev. E 67, 066102 (2003). 